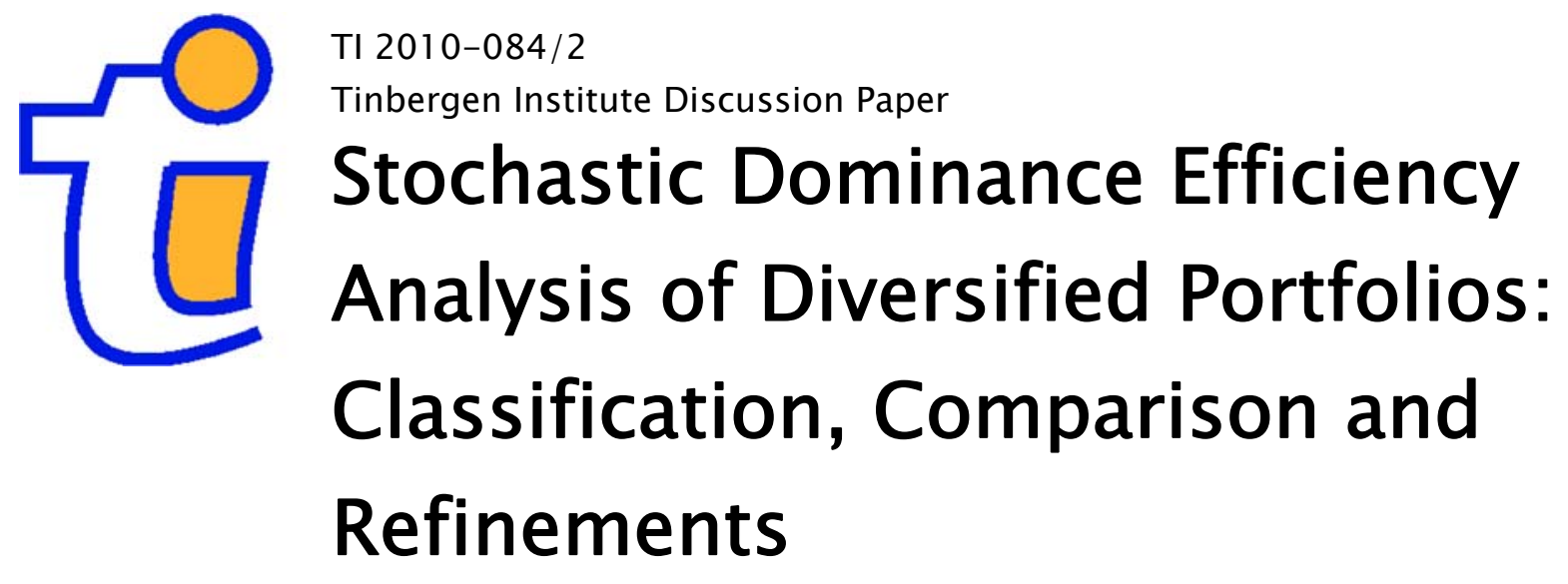

Andrey Lizyayev 


\section{Tinbergen Institute}

The Tinbergen Institute is the institute for economic research of the Erasmus Universiteit Rotterdam, Universiteit van Amsterdam, and Vrije Universiteit Amsterdam.

Tinbergen Institute Amsterdam

Roetersstraat 31

1018 WB Amsterdam

The Netherlands

Tel.: +31(0)205513500

Fax: $+31(0) 205513555$

Tinbergen Institute Rotterdam

Burg. Oudlaan 50

3062 PA Rotterdam

The Netherlands

Tel.: + $31(0) 104088900$

Fax: $+31(0) 104089031$

Most TI discussion papers can be downloaded at http://www.tinbergen.nl. 


\title{
Stochastic Dominance Efficiency Analysis of Diversified Portfolios: \\ Classification, Comparison and Refinements
}

\author{
Andrey Lizyayev*
}

July 26,2010

\begin{abstract}
For more than three decades, empirical analysis of stochastic dominance was restricted to settings with mutually exclusive choice alternatives. In recent years, a number of methods for testing efficiency of diversified portfolios have emerged, which can be classified into three main categories: 1) majorization, 2) revealed preference and 3) distribution-based approaches. Unfortunately, some of these schools of thought are developing independently, with little interaction or crossreferencing among them. Moreover, the methods differ in terms of their objectives, the information content of the results and their computational complexity. As a result, the relative merits of alternative approaches are difficult to compare. This paper presents the first systematic review of all three approaches in a unified methodological framework. We examine the main developments in this emerging literature, critically evaluating the advantages and disadvantages of the alternative approaches. We also point out some misleading arguments and propose corrections and improvements to some of the methods considered.
\end{abstract}

Key words: Stochastic Dominance, Portfolio Efficiency, Algorithms.

JEL Classification: G11, C63.

*Erasmus University Rotterdam, P. O. Box 1738, 3000 DR Rotterdam, The Netherlands. E-mail: lizyayev@ese.eur.nl 


\section{Introduction}

The relation of Stochastic Dominance, introduced in mathematics by Mann and Whitney (1947) and Lehmann (1955), and in economics by Quirk and Saposnik (1962), Hadar and Russell (1969), Hanoch and Levy (1969) and Rothschild and Stiglitz (1970, 1971), is broadly applied in financial decision making under uncertainty (see, for example, Bawa (1982) and Levy $(1992,2006)$ for a survey and references). For more than three decades, empirical analysis of stochastic dominance was restricted to settings with mutually exclusive choice alternatives, appropriate for comparison of income distributions or crop yields in agriculture, for example. These methods include various mean-risk models (see e.g. Hogan and Warren (1972), Ang (1975), Shalit and Yizhaki (1984)) and direct pairwise efficient comparison of distribution functions (Hadar and Russell (1969), Bawa et al. (1979), Aboudi and Thon (1994), Anderson (1996), Annaert et al (2009), among many others). However, pairwise comparison algorithms are insufficient for identifying dominating portfolios from an infinite set of diversified portfolios, which is a typical setting in finance. Levy (1992) emphasizes this problem by stating:

"Ironically, the main drawback of the SD framework is found in the area of finance where it is most intensively used, namely, in choosing the efficient diversification strategies. This is because as yet there is no way to find the SD efficient set of diversification strategies as prevailed by the $\mathrm{M}-\mathrm{V}$ framework. Therefore, the next important contribution in this area will probably be in this direction".

Some authors introduced other SD-related concepts, such as convex SD (Fishburn (1974)) and marginal conditional SD (Shalit and Yitzhaki (1994)). Such methods can only provide a necessary condition for stochastic dominance efficiency when the portfolio possibilities set has a particular structure, but not in general.

In recent years, stochastic dominance literature has developed a number of methods for analyzing efficiency of diversified portfolios, following the works of Kuosmanen (2001-WP, 2004), Post (2003) and Dentcheva and Ruszczyński (2003). Although Dybvig and Ross (1982) propose SSD efficiency criteria that can be developed into an SSD efficiency test with diversification (such as in Lizyayev (2009)), they only provide a useful idea, but not an explicit algorithm. 
The first authors to address stochastic dominance relative to an infinite set of choice alternatives after Dybvig and Ross (1982) were Ogryczak and Ruszczyński $(1999,2001,2002)$ in their mean-risk models. Ogryczak and Ruszczyński (1999) proposed an optimization problem that identified mean-risk efficient frontiers of stochastically nondominated portfolios, and extended it to higher-order semideviations in Ogryczak and Ruszczyński (2001). Subsequently, Ruszczyński and Vanderbei (2003) have explicitly formulated the frontier identification problem for portfolio weights, and suggested an efficient parametric optimization. Although mean-risk models cannot generally solve the problem of identifying whether a given portfolio is SD efficient (which is the formulation usually employed in asset pricing and investment management), they can be used as a necessary condition for SSD efficiency.

To our knowledge, Dentcheva and Ruszczyński (2003) and Kuosmanen (2004) independently developed the first algorithms to identify a portfolio that dominates a given benchmark among an infinite number of diversified portfolios by solving a finite dimensional optimization problem. A preliminary version of Kuosmanen's test appeared in Kuosmanen (2001-WP) working paper. Meanwhile, Post (2003) developed an alternative test which is simpler and computationally less demanding, but does not generally produce a dominating portfolio. Dentcheva and Ruszczyński (2003) introduced an optimization model with stochastic dominance constraints and developed this model further in Dentcheva and Ruszczyński (2006-b) and Rudolf and Ruszczyński (2008). Although this model has an arbitrary objective function and in this respect is more general, we will focus on its use in the most frequently applied setting in finance, namely: identifying the SD efficiency of a given portfolio relative to a diversified portfolio possibilities set. Dentcheva and Ruszczyński (2006-a) introduced inverse stochastic dominance constraints, which were later employed in Kopa and Chovanec's (2008) refined method for testing stochastic dominance efficiency.

The literature of stochastic dominance currently spans a number of alternative methods. To structure this literature, we propose to classify the present approaches into three categories: 1) majorization, 2 ) revealed preference and 3) distribution-based approaches. These approaches differ in their objectives, the information content of the results, and their computational complexity. Unfortunately, some of these schools of thought are developing independently, with little in- 
teraction or cross-reference to the other schools and as a result the advantages and disadvantages of alternative approaches have not been compared in a fair and systematic fashion. The proponents of each method have a natural tendency to exaggerate the advantages of their favorite method and overlook the advantages of their competitors'.

This paper presents the first systematic attempt to bring all three approaches under the common umbrella of a unified methodological framework. We will examine the main developments in this emerging literature, critically evaluating the advantages and disadvantages of the alternative approaches using a number of objective criteria. We will also point out some misleading arguments in this literature and propose corrections and improvements to some of the methods considered.

The paper is organized as follows. In Section 2 we define the basic general concepts related to stochastic dominance efficiency and state some common assumptions. Since most of the methods are applied to the second order stochastic dominance (SSD), where the efficiency test becomes a linear program ${ }^{1}$, we classify, analyze and compare the most important SSD efficiency algorithms to date in Section 3. To keep such comparative analysis objective, we use a unified framework of Section 2 and adjust each of the methods considered in such a way that they solve the same standardized problem which is commonly and frequently used in practice. In Section 4 we consider some extensions to the standardized framework such as first order stochastic dominance (FSD) and unbounded short sales, and analyze the extent to which the existing methods can tackle those modifications. Finally, Section 5 gives some concluding remarks and finalizes the paper.

\footnotetext{
${ }^{1}$ Some authors use more demanding non-linear programs (such as Linton $(2005,2010)$ and the iterative quadratic program of Post and Versijp (2007) which, in addition to the efficiency outcome, also provide statistical significance scores under some assumptions. Since such programs do not produce a dominated portfolio and are considerably more computationally demanding, we will omit them from our analysis. As statistical significance scores can be more naturally obtained via non-parametric bootstrapping procedures in the framework of this Chapter, we will focus on SSD efficiency tests which are more practical in terms of the computational complexity and the information content of the result.
} 


\section{Unified framework}

As a first step towards bringing alternative approaches under a common umbrella, we need a general framework into which all alternative methods can naturally fit. It is the purpose of this section to describe such a framework. We should note that some of the methods reviewed in the subsequent sections do not necessarily require all of the assumptions imposed in this section. In the interest of clarity, however, we will review all methods from the perspective of the unified framework, duly noting the possible extensions as we proceed.

A canonical model of investment decision making in a static setting can be described as follows. There are $n$ marketed assets, whose returns may vary across different states of nature. From $m$ possible states, one state is randomly drawn as the realized state. Returns of assets in $m$ alternative states of nature are described by $m$-by- $n$ matrix $X$. If a riskless asset is available in the market, we can include it as one column of $X$ (a column with equal components). Naturally, all asset returns are assumed to be linearly independent, which implies that $X^{\top} X$ is positive definite. Note that there is no uncertainty about the return matrix $X$; the investors' risk arises from the random realization of one out of $m$ possible states. Without loss of generality, we assume all states to be equally likely. ${ }^{2}$

Investors may diversify between available assets. We shall use $\lambda \in$ $\mathbb{R}^{n}$ for a vector of portfolio weights. The portfolio possibilities set (assuming away short sales) is

$$
\Lambda=\left\{\lambda \in \mathbb{R}^{n}: \lambda^{\top} e=1, \lambda \geq 0\right\}^{3},
$$

and the set of all available allocations is

$$
M_{X}=\left\{x \in \mathbb{R}^{m}: x=X \lambda, \lambda \in \Lambda\right\} .
$$

Each investor has a von Neuman-Morgenstern utility function $u \in$ $U=\{u: \mathbb{R} \rightarrow \mathbb{R}\}$ which depends on his final wealth at the end of the

\footnotetext{
${ }^{2}$ States with different probabilities can be dealt with by a linear transformation of decision variables so that the resulting program will be equivalent to the one with equally probable states; see Dybvig and Ross (1982) for details.

${ }^{3}$ Unless otherwise stated, we will consider the PPS with short sales restricted. Nonetheless some other restrictions on portfolio possibilities may apply in practice; moreover the use of some methods can be particularly advantageous for certain classes of PPS, as will be shown in subsequent sections.
} 
holding period. As shown in Pratt (1964), investors' non-satiation and risk attitude can be modeled via the first and second derivative of $u$, respectively. The class of increasing utility functions which represents all non-satiable investors is denoted by $U_{1}$, and the class of increasing and concave utility functions is denoted by $U_{2}$ and represents all nonsatiable and risk-averse investors. Formally,

$$
U_{1} \equiv\left\{u: \mathbb{R} \rightarrow \mathbb{R} \quad \text { s.t. } u^{\prime}(t) \geq 0, \forall t\right\}
$$

and

$$
U_{2} \equiv\left\{u: \mathbb{R} \rightarrow \mathbb{R} \quad \text { s.t. } u^{\prime}(t) \geq 0, \text { and } u^{\prime \prime}(t) \leq 0, \forall t\right\} .
$$

Due to the uncertainty about which state of the world will occur, investors seek to maximize their expected utility. Portfolio $\tau \in \Lambda$ is the optimal choice for an investor with utility $u \in U$ if and only if

$$
\mathbb{E} u(X \tau)=\sup _{\lambda \in \Lambda} \mathbb{E} u(X \lambda),
$$

where $\mathbb{E}$ denotes the expected value operator. Since all states are equally likely by assumption, equation (1) can be equivalently stated as

$$
\sum_{i=1}^{m} u\left(x^{i} \tau\right)=\sup _{\lambda \in \Lambda} \sum_{i=1}^{m} u\left(x^{i} \lambda\right) .
$$

Observing a given portfolio $\tau$, our purpose is to evaluate whether $\tau$ is the optimal choice for a group of investors. Since the investors' utility functions are unknown, we focus on broad classes of economically meaningful utility functions, $U_{1}$ and $U_{2}$. To this end, the following definitions prove useful.

Definition 1 (dominance). Portfolio $\lambda \in \Lambda$ dominates portfolio $\tau \in \Lambda$ by First Order Stochastic Dominance, further FSD (by Second Order Stochastic Dominance, further $\boldsymbol{S S D}$ ) if and only if for all utility functions $u \in U_{1}\left(u \in U_{2}\right)$

$$
\sum_{i=1}^{m} u\left(x^{i} \lambda\right) \geq \sum_{i=1}^{m} u\left(x^{i} \tau\right),
$$

with a strict inequality for at least one $u \in U_{1}\left(u \in U_{2}\right)$. 
Definition 2 (super-dominance). Portfolio $\lambda \in \Lambda$ super-dominates portfolio $\tau \in \Lambda$ by FSD (SSD) if and only if for all strictly increasing utility functions $u \in U_{1}\left(u \in U_{2}\right)$

$$
\sum_{i=1}^{m} u\left(x^{i} \lambda\right)>\sum_{i=1}^{m} u\left(x^{i} \tau\right),
$$

Definition 1 is standard in the stochastic dominance literature. The notion of super-dominance is a new term that we have coined for the definition first proposed by Post (2003). Note that superdominance implies dominance, but the reverse is not true. For example, if $\tau$ is a mean-preserving spread of portfolio $\lambda$, then $\tau$ dominates $\lambda$ by SSD, but it does not super-dominate it.

Definitions 1 and 2 can be stated analogously for any given class of utility functions $U$. Although $U_{1}$ and $U_{2}$ are the most frequently used, some authors developed tests for refined utility classes, e.g. modeling increasing relative and decreasing absolute risk aversion,such as Vickson $(1975,1977)$ and Lizyayev (2009).

Using Definitions 1 and 2, the notions of portfolio efficiency and optimality are defined as follows:

Definition 3 (weak efficiency). Portfolio $\tau \in \Lambda$ is weakly FSD (SSD) efficient if and only if there does not exist another portfolio $\lambda \in \Lambda$ that super-dominates $\tau$ in the sense of Definition 2.

Definition 4 (strong efficiency). Portfolio $\tau \in \Lambda$ is strongly FSD (SSD) efficient if and only if there does not exist another portfolio $\lambda \in \Lambda$ that dominates $\tau$ in the sense of Definition 1.

Definition 5 (optimality). Portfolio $\tau \in \Lambda$ is FSD (SSD) optimal if and only if there exists a strictly increasing $u \in U_{1}\left(u \in U_{2}\right)$ for which $\tau$ is the optimal portfolio choice, that is,

$$
\sum_{i=1}^{m} u\left(x^{i} \tau\right)>\sum_{i=1}^{m} u\left(x^{i} \lambda\right), \text { for all } \lambda \in \Lambda \backslash\{\tau\} .
$$

There exist alternative equivalent definitions of stochastic dominance which we state below.

Definition 6. Allocation $x \in M_{X}$ with cumulative distribution function $(C D F) F_{X}(z)$ dominates allocation $y \in M_{X}$ having $C D F F_{Y}(z)$ by FSD (SSD) if and only if

$$
F_{X}(z) \leq F_{Y}(z)\left(F_{X}^{(2)}(z) \leq F_{Y}^{(2)}(z)\right),
$$


for all $z$, with a strict inequality for at least one $z$,

where $F_{X}^{(2)}(z)$ is defined as $F_{X}^{(2)}(z) \equiv \int_{-\infty}^{z} F_{X}(t) d t=\mathbb{E}(\max \{z-X, 0\})$.

Due to the latter representation $F_{X}^{(2)}(z)$ is also called the expected shortfall of $X$. Similarly, SD relation can be equivalently formulated in terms of (integrated) inverted CDF (quantiles) as follows. Condition (5) is equivalent to

$F_{X}^{-1}(q) \geq F_{Y}^{-1}(q)\left(F_{X}^{-2}(q) \equiv \int_{0}^{q} F_{X}^{-1}(v) d v \geq \int_{0}^{q} F_{Y}^{-1}(v) d v \equiv F_{X}^{-2}(q)\right)$,

for all $q \in[0,1]$.

SSD condition (6) can also be expressed in terms of conditional value at risk $(\mathrm{CVaR})$ which is related to $F_{X}^{-2}(q)$ (see Rockafellar and Uryasev (2002)) as

$$
F_{X}^{-2}(q)=-q \mathrm{CVaR}_{1-q}(-X), q \in(0,1) .
$$

Definition 7. Allocation $x \in M_{X}$ dominates allocation $y \in M_{X}$ by FSD (SSD) if and only if

$$
\exists P \in \Pi(\exists W \in \Xi): x \geq P y(x \geq W y),
$$

where $\Pi$ is the class of permutation matrices:

$$
\Pi=\left\{\left[w_{i j}\right]_{m \times m}: w_{i j} \in\{0,1\}, \sum_{i=1}^{m} w_{i j}=\sum_{j=1}^{m} w_{i j}=1, i, j=1, \ldots, m\right\}
$$

and $\Xi$ is the class of doubly stochastic matrices:

$\Xi=\left\{\left[w_{i j}\right]_{m \times m}: 0 \leq w_{i j} \leq 1, \sum_{i=1}^{m} w_{i j}=\sum_{j=1}^{m} w_{i j}=1, i, j=1, \ldots, m\right\}$.

Definitions 1, 6 and 7 are known to be equivalent. The equivalence of definitions 1 and 6 is easy to prove by changing variables in the integration of Definition 6. For the equivalence of Definition 7 see Hardy et al (1934), Hadar and Russel (1969) and Marshall and Olkin (1979). 
For the sake of brevity we will sometimes refer (with a slight abuse of notation) to an allocation by the corresponding portfolio, for instance by stating that portfolio $\tau \in \Lambda$ dominates allocation $y \in M_{X}$ we mean that $X \tau$ dominates $y$.

The difference between the FSD and SSD efficiency arises from the assumption of risk aversion: SSD assumes risk aversion, whereas FSD does not. In the case of SSD, the optimality and efficiency definitions (2) and (3) are equivalent if the portfolio possibilities set $\Lambda$ is convex. However, FSD optimality is only a necessary condition for FSD efficiency, even with a convex $\Lambda$.

Restrictions on the set of utility functions strongly affect the computational complexity of a test, as will be demonstrated below. The computational burden becomes particularly restrictive when it comes to bootstrapping and statistical inference. To assess whether the outcome of a test is statistically significant (and cannot be attributed solely to chance), one needs to simulate a large number of new data sets of asset returns generated by the same distribution as the original, and further to run the same efficiency test on all those samples. With current computing power and the usual dimensionality of the data, only certain types of optimization programs can be tackled within a reasonable time, such as linear or quadratic programs. Mixed integer linear programs (which FSD efficiency tests are in essence) are far too demanding for any rigorous bootstrapping techniques. For that reason, and because the vast majority of the tests used in practice are focused on second order stochastic dominance efficiency, we will analyze them in detail below.

\section{Second Order (SSD) Efficiency}

The extensive literature which suggests SSD efficiency algorithms can be grouped into three main categories: 1) majorization, 2) revealed preference, and 3) distribution-based approaches. The first category is based on optimality conditions in the space of returns given in Definition 7; the second on Lagrangean conditions for the marginal utility rationalizing a given portfolio in accordance with Definition 5, and the last on various equivalent criteria of SD efficiency formulated directly on cumulative distribution functions of underlying portfolios as in Definition 6. Although the categories above are not mutually exclusive (e.g. the dual formulation to distribution-based approach has 
a revealed preference interpretation), most of the methods are most frequently used either in their primal or dual form, which we will take as the basis for our classification. In this paper we attempt to cover the most efficient methods of each school of thought.

To characterize and compare all the methods in a fair and systematic fashion we would like to point out the criteria an SSD efficiency test should fulfill. Clearly, the primary goal of every method should be to identify whether a given portfolio is efficient relative to a given convex portfolio possibilities set in the sense of Definition $4 .{ }^{4}$ The methods therefore should provide necessary and sufficient conditions for such efficiency. In cases when the subject portfolio is inefficient, one would like to have a measure indicating the degree of its inefficiency. A natural choice for such a measure could be the highest possible difference in mean returns between the subject portfolio and an efficient marketed portfolio that dominates it. If there is a dominating portfolio with the same mean return as the subject portfolio but with a tighter spread around the risk-free asset (this dominance is self-evident and formally in accordance with Definition 4), one would like to incorporate the maximal feasible spread into the measure of inefficiency as well. For that reason, it is desirable that when a given portfolio is inefficient, an efficiency test identifies a dominating portfolio that is marketed and SSD efficient itself. Another advantage would be if the method could be split into some sequential subtests that are less computationally demanding, so that one could identify inefficiency at an earlier stage based on some necessary conditions, in which case running the rest of the test would be unnecessary. Finally, the ability of SSD tests to be easily generalized to FSD efficiency testing would also be of value.

\subsection{Revealed Preference Approach}

The revealed preference approach has its roots in Afriat's (1967) celebrated theorem. Analogous to Afriat's test of rational consumer behavior $^{5}$, SSD efficiency can be tested based on the first order optimality conditions for the utility function which, provided that such

\footnotetext{
${ }^{4}$ Although occasionally we will distinguish the weak efficiency in the sense of Definition 3 , we adapt the commonly accepted SSD efficiency given by Definition 4 throughout, and unless otherwise stated, SSD efficiency will refer to this strong definition.

${ }^{5}$ Varian (1983) has applied Afriat's approach to testing rationality of investor behavior in a somewhat different setting than the one considered in this paper.
} 
function exists, would rationalize the subject portfolio, in accordance with Definition 5 and the fact that SSD optimality is equivalent to SSD efficiency if the portfolio possibilities set is convex. The general idea of the revealed preference approach is to try to find marginal utilities $\beta$ for some well-behaved von Neumann-Morgenstern utility function for which the evaluated portfolio $y \in M_{X}$ is the optimal solution maximizing its expected value. If such marginal utilities $\beta$ exist, then the evaluated portfolio is literally "revealed optimal", at least for some hypothetical decision maker with rational preferences. If such marginal utilities do not exist, then the evaluated portfolio $y$ is SSD inefficient.

While the marginal conditional stochastic dominance introduced in Shalit and Yitzhaki (1994) can, like some other earlier methods, formally be assigned to this category, it uses different settings in which the subject portfolio is tested relative to a set of vertices of a portfolio possibilities set. This test is computationally less demanding but can only be used as a first-stage necessary pre-processing test for our framework, as it can not generally identify SSD efficiency in the case of full diversification. Marginal conditional formulation also appears as duality results in the distribution-based methods, such as Dentcheva and Ruszczyński (2006-a, 2006-b) and Rudolf and Ruszczyński (2008). However, the primal distribution-based method appears to be computationally competitive relative to its dual linear programming formulations. Therefore we will classify these tests as distribution-based and will cover them below in a separate sub-section.

Post (2003) formulates the following revealed preference test for SSD efficiency of a given marketed portfolio $y \in M_{X}$ :

$$
\begin{gathered}
\xi(y)=\min \theta \\
\text { s.t. } \frac{1}{m} \sum_{t=1}^{m} \beta_{t}\left(y_{t}-X_{t i}\right)+\theta \geq 0, \quad i=1, \ldots, n \\
\beta_{1} \geq \beta_{2} \geq \cdots \geq \beta_{m}=1 \\
\lambda \in \Lambda \\
\theta \text { free }
\end{gathered}
$$

Parameters $\beta_{t}$ can be interpreted as Afriat numbers, which represent the marginal von Neumann-Morgenstern utility of some rational decision maker in state $t$. If the optimal solution to (7) satisfies $\xi^{*}(y)=0$, then the evaluated portfolio is an optimal solution that maximizes expected utility to some rational risk-averse decision 
maker. Thus $\xi^{*}(y)=0$ is a necessary and sufficient condition for weak SSD efficiency of $y$ (given in Definition 3) and, as Kuosmanen (2004) notes, only a necessary condition for the strong SSD efficiency (in the sense of Definition 4).

Kuosmanen (2004, Sec. 4.4) derives a similar test based on the idea of separating hyperplanes. Both methods are only capable of determining the efficiency status of a given portfolio; they do not generally produce a dominating portfolio. The major advantage of the methods is their computational simplicity: (7) is a linear program with $m+1$ variable and $n+m$ constraints.

Post (2003) also derives a dual formulation to (7) as follows.

$$
\begin{aligned}
\psi(y) & =\max s_{m} \\
\text { s.t. } \frac{1}{m} \sum_{i=1}^{k}\left(x^{i} \lambda-y_{i}\right) & =s_{k}, \quad k=1, \ldots, m \\
\lambda & \in \Lambda \\
s & \in \mathbb{R}_{+}^{m}
\end{aligned}
$$

If the optimal solution to (8) is $\psi^{*}=0$, then $y$ is weakly SSD efficient. Although the optimal portfolio $\lambda^{*}$ has an intuitive interpretation as the portfolio with the largest increase in the mean return, it does not necessarily dominate $y$. To see this, consider (8) for $y=[1,4], x^{1}=[9,0], x^{2}=[0,2], x^{3}=y$. Running the tests yields $\xi^{*}(y)=\psi^{*}(y)=2$ which correctly identifies SSD inefficiency of $y$, however $X \lambda^{*}=x^{1}=[9,0]$, even though $x^{1}$ does not dominate $y$.

Post (2008) has extended the SSD test for weak efficiency to the standard case of strong efficiency (Definition 4) by simply changing the objective function of (8) from $s_{m}$ to the sum $s^{\top} e$, obtained $s^{\top} e=0$ as the necessary and sufficient condition for the strong SSD efficiency and shown that the subject portfolio $y$ is always SSD dominated by a linear combination of $X \lambda^{*}$ and $y$. However, a dominating portfolio obtained thus does not necessarily have the highest mean return among all dominating portfolios, and therefore is not suitable as a benchmark for efficiency gauging. Further, the dominating portfolio is not necessarily SSD efficient even in the sense of weak SSD efficiency (Definition 3). 


\subsection{Majorization Approach}

The majorization approach is based on Definition 7, which originates in the mathematical literature on stochastic dominance, where the concept appeared as stochastic ordering. The first majorization-based test in economic literature appeared in Kuosmanen (2001-WP) and was further developed in Kuosmanen (2004).

Kuosmanen (2004) splits SSD efficiency test into necessary and sufficient subtests. The necessary test reads ${ }^{6}$

$$
\begin{aligned}
\theta_{2}^{N}(y) & =\max _{\lambda, W}(X \lambda-y)^{\top} e \\
\text { s.t. } X \lambda & \geq W y \\
W & \in \Xi \\
\lambda & \in \Lambda
\end{aligned}
$$

Comparing (9) with Post's (2008) dual (8) reveals that the two problems are structurally similar, except for the doubly stochastic matrix $W$ included in (9). Post (2008) sorts the asset returns in ascending order with respect to $y$, whereas Kuosmanen did not utilize the prior ordering. As a result, the optimal portfolio $\lambda^{*}$ of (9) always SSD dominates $y$ when the latter is inefficient (provided $W^{*}$ is not a permutation matrix), contrary to (8).

Kuosmanen (2004) shows that $\theta_{2}^{N}=0$ is a necessary condition for the strong SSD efficiency of portfolio $y$. Note, that $\theta_{2}^{N} / m$ can be intuitively used as an inefficiency measure that indicates the difference between the mean return of the dominating portfolio $\lambda^{*}$ with the highest mean return and the expected return of $y$. Another possibility considered by Kuosmanen (2004) is to gauge efficiency by using the minimum risk-free premium that needs to be added to $y$ to make it SSD efficient. While such a measure can be intuitive for gauging inefficiency loss, it cannot provide a necessary SSD efficiency condition analogous to (9). The same is true for the more general directional distance function formulated in Kuosmanen (2007).

Kuosmanen (2007) derives the dual formulation to (9), which can be expressed as

\footnotetext{
${ }^{6}$ Kuosmanen (2004) formulates (9) with $X$ augmented by $y$, as it can happen that $y \notin M_{X}$ is SSD efficient, but is dominated by a linear combination of a marketed portfolio and itself. We omit this augmentation here for the sake of comparability with the other methods.
} 


$$
\begin{aligned}
\xi^{D}(y) & =\min _{\beta, \theta, a, b} \theta-\left(a^{\top} e+b^{\top} e\right) \\
\text { s.t. } \theta e & \geq X^{\top} \beta \\
\beta_{s} y_{t} & \geq y_{t}+a_{t}+b_{s}, \quad \forall s, t=1, \ldots, m \\
\beta & \geq e \\
\theta & \in \mathbb{R}, \quad a, b, \beta \in \mathbb{R}^{m}
\end{aligned}
$$

Clearly, the dual program (10) is similar to (9) in terms of the computational complexity. However, (10) is less intuitive and its coefficients are difficult to interpret. Moreover, it is unclear if (10) can be generalized to a sufficient test for SSD efficiency of $y$ in a straightforward way. For that reason we shall focus on the primal formulation (9) for which Kuosmanen (2004) proposed the following sufficient test statistic.

$$
\begin{aligned}
\theta_{2}^{S}(y) & =\min \sum_{i=1}^{m} \sum_{j=1}^{m}\left(s_{i j}^{+}+s_{i j}^{-}\right) \\
\text {s.t. } X \lambda & =W y \\
s_{i j}^{+}+s_{i j}^{-} & =w_{i j}-\frac{1}{2}, \quad i, j=1, \ldots, m \\
s_{i j}^{+}+s_{i j}^{-} & \geq 0, \quad i, j=1, \ldots, m \\
W & \in \Xi, \lambda \in \Lambda
\end{aligned}
$$

Program (11) minimizes $\sum_{i=1}^{m} \sum_{j=1}^{m}\left|w_{i j}-\frac{1}{2}\right|$. The underlying idea lies in finding a marketed mean-preserving spread of $y$ that is as close to the risk free ray as possible. The non-existence of any such $X \lambda^{*} \neq y$ would then suffice for SSD efficiency of $y$. Kuosmanen (2004) proposes the theoretical maximum of the test statistic as a sufficient condition ${ }^{7}$ :

$$
\theta_{2}^{S}(y)=\frac{m^{2}}{2}-\sum_{k=2}^{m} k d_{0 k}
$$

where $d_{0 k}$ is the number of $k$-way ties.

\footnotetext{
${ }^{7}$ Kuosmanen (2004) defines $\theta_{2}^{S}(y)$ as $\frac{m^{2}}{2}-\sum_{k=1}^{m} k d_{0 k}$; however he clearly meant (12). Moreover, the summation $\sum_{k=1}^{m} k d_{0 k}$ equals $m$, since it counts all $m$ elements of $y$ precisely once.
} 
Although the optimal $X \lambda^{*}$ from (11) always SSD dominates $y$ (provided the two portfolios are distinct) it may not be SSD efficient. To see this, consider the following example. Suppose we test portfolio $y=[2,0,10]$ and both $x^{1}=[6,5,1]$ and $x^{2}=[4,4,4]$ are marketed. If $\theta_{2}^{S}(y)=3 / 2$, the program (11) may have chosen $x^{1}$ with $W_{1}^{*}=\frac{1}{2}\left[\begin{array}{lll}1 & 0 & 1 \\ 0 & 1 & 1 \\ 1 & 1 & 0\end{array}\right]$, however $x^{2}$ may have been chosen as well with $W_{2}^{*}=\frac{1}{3}\left[\begin{array}{lll}1 & 1 & 1 \\ 1 & 1 & 1 \\ 1 & 1 & 1\end{array}\right]$, since $W_{1}^{*}$ and $W_{2}^{*}$ give the same value of statistic $\theta_{2}^{S}(y)$. Therefore, if (11) picks $x^{1}$, it dominates $y$ but is not SSD efficient.

If the efficiency of the dominating portfolio is required, one can use the following quadratic programming extension of (11).

$$
\begin{aligned}
\theta_{2}^{R}(y) & =\min \lambda^{\top} X^{\top} X \lambda-y^{\top} y \\
\text { s.t. } X \lambda & =W y \\
W & \in \Xi \\
\lambda & \in \Lambda
\end{aligned}
$$

Note that (13) minimizes the second moment of $X \lambda$ which is equivalent to minimizing the Euclidian distance from $y$ to the risk free asset $e \cdot \mathbb{E} y$. We can prove the following

Proposition 1. Suppose $\theta_{2}^{N}(y)=0$. Portfolio y is SSD efficient with respect to $\Lambda$ if and only if $\theta_{2}^{R}(y)=0$. Moreover, $\lambda^{*}$ from (13) is $S S D$ efficient and, if $\theta_{2}^{R}(y) \neq 0$, dominates $y$.

Proof. It follows from the majorization theory (see Marshall and Olkin, $1979)$ that if for some $W \in \Xi, W y$ is not a permutation of $y$, then $y^{\top} W^{\top} W y<y^{\top} y$. Given that $X=W y$, the objective $\lambda^{\top} X^{\top} X \lambda-$ $y^{\top} y=y^{\top} W^{\top} W y-y^{\top} y=y^{\top}\left(W^{\top} W-I_{m}\right) y \leq 0$. Therefore, $\theta_{2}^{R}(y)=0$ implies $W^{*} y=P y$, for some permutation matrix $P \in \Pi$, and thus $y$ is efficient. Similarly, if $\theta_{2}^{R}(y)<0$, then $y$ is dominated by $W^{*} y$, so $y$ is SSD inefficient. The efficiency of $X \lambda^{*}$ follows from the fact that the existence of a strictly dominating portfolio $X \tau=W X \lambda^{*}$ would contradict the optimality of $X \lambda^{*}$ in (13).

Summarizing, we can characterize the method as follows. The necessity test (9) is a linear program with $m^{2}+n$ variables, $m^{2}+m$ inequality and $2 m$ equality constraints. Program (11) with $3 m^{2}+n$ variables, $m^{2}+3 m$ equality and $3 m^{2}$ inequality constraints is a sufficient test for SSD efficiency of $y$, but the optimal portfolio itself may 
not be SSD efficient. An alternative sufficient condition is given by Proposition 1 that does generate an SSD efficient dominating portfolio $W^{*} y$ as a byproduct. This test is based on quadratic program (13) with $m^{2}+n$ variables (of which $n$ enter the objective), $3 m$ linear equality and $m^{2}$ linear inequality constraints.

Based on the general theoretical result in Strassen (1965), Luedtke (2008) recently developed the majorization test (9) further by explicitly including the probabilities of the states (which are assumed equal in (9)) and suggested a branching heuristic for solving the method. His linear programming formulation, however, closely resembles (9), particularly in terms of the computational complexity.

\subsection{Distribution-Based Approach}

This group of methods is based on Definition 6 and usually employs equivalent definitions involving various modifications of the cumulative distribution function and its inverse, such as integrated (inverted) $\mathrm{CDF}$, quantiles and conditional value at risk.

Dentcheva and Ruszczyński (2003) introduced the following linear program with distribution-based stochastic dominance constraints.

$$
\begin{aligned}
\max f(\lambda) & =\mathbb{E}(X \lambda) \\
\text { s.t. } \sum_{k=1}^{n} x_{i k} \lambda_{k}+s_{i j} & \geq y_{j}, \quad i, j=1, \ldots, m \\
\sum_{i=1}^{m} s_{i j} & \leq m v_{j}, \quad j=1, \ldots, m \\
s_{i j} & \geq 0, \quad i, j=1, \ldots, m \\
\lambda & \in \Lambda
\end{aligned}
$$

where $v_{j} \equiv \mathbb{E}\left[\left(y_{j}-y\right)_{+}\right]=F_{Y}^{2}\left(y_{j}\right)$ is the expected shortfall of $y$.

The constraints in (14) basically ensure that $\mathbb{E}\left[(a-X \lambda)_{+}\right] \leq \mathbb{E}[(a-$ $\left.y)_{+}\right], \forall a$, which by Definition 6 is equivalent to the SSD dominance of $X \lambda$ over $y$ (see Dentcheva and Ruszczyński (2003, 2006-b) for more details).

Rudolf and Ruszczyński (2008) elaborated on this method, suggesting two alternative implementations of (14): a primal cutting plane method and a dual column generation method. However, they concluded that the dual method proved to be practically prohibitive 
for this problem (compared to a straightforward simplex implementation of (14)). The primal method was shown to outperform the simplex on their data set. However it is not clear if such performance can be generalized on an arbitrary data set; the method may require a factorial number of iterations in the worst case scenario.

Just like Kuosmanen's (2004) test (9), program (14) always produces a weakly SSD efficient dominating portfolio $\lambda^{*}$ which may not be (strongly) SSD efficient (this may happen when (14) has multiple solutions). To overcome this, consider the following sufficiency test statistic.

$$
\begin{aligned}
& \theta^{R}(y)=\sum_{i=1}^{m}\left(y_{i}-\mathbb{E}(y)\right)^{2}-\min \sum_{i=1}^{m}\left(\sum_{j=1}^{n} x_{i j} \lambda_{j}-\mathbb{E}(y)\right)^{2} \\
& \text { s.t. } \sum_{k=1}^{n} x_{i k} \lambda_{k}+s_{i j} \geq y_{j}, \quad i, j=1, \ldots, m \\
& \sum_{i=1}^{m} s_{i j} \leq m v_{j}, \quad j=1, \ldots, m \\
& s_{i j} \geq 0, \quad i, j=1, \ldots, m \\
& \lambda \in \Lambda
\end{aligned}
$$

Proposition 2. Let $x^{*}=X \lambda^{*}$ be a solution of (14) for a given portfolio $y \in M_{X}$. Determine $\theta^{R}\left(x^{*}\right)$ by solving (15) and denote the optimal solution by $z^{*}$. Portfolio $y$ is SSD inefficient if and only if

$$
\mathbb{E}\left(x^{*}\right)-\mathbb{E}(y)+\theta^{R}\left(x^{*}\right)>0
$$

Moreover, (16) also implies that $y$ is dominated by $z^{*}$ which is SSD efficient.

Proof. First note, that solution $z^{*}$ to (15) is unique, due to the strict convexity of the objective function in (15) and linear independence of returns. Due to the dominance restrictions imposed in (15), $z^{*}$ is SSD efficient. Since (16) holds if and only if $z^{*}$ and $y$ are distinct, the result follows.

Program (14) is closely related to Kuosmanen's necessary test (9) in terms of the information content of the result. Both methods can identify a necessary and sufficient condition for the weak SSD efficiency (Definition 3), but only a necessary condition for the standard 
SSD efficiency. The optimal reference portfolio $X \lambda^{*}$ dominates $y$ and is itself weakly SSD efficient. If several dominating portfolios of equal mean are available, both methods may select a dominating portfolio that is not (strongly) SSD efficient. Moreover, the two methods are following the same principle: to maximize the mean return among all available portfolios that dominate $y$ and hence both can be used for inefficiency gauging. The only difference is that Kuosmanen (2004) exploits a majorization-based, and Dentcheva and Ruszczyński distribution-based dominance criteria. Test (14) is a linear program with $m^{2}+n$ variables and $2 m^{2}+m$ constraints which is computationally heavier than Kuosmanen's necessary test (9), but lighter than his sufficiency test (11). Combined with (14), test (15) produces an SSD efficient dominating portfolio when the subject portfolio is inefficient.

Another distribution-based test recently published in Kopa and Chovanec (2008) employs the conditional value at risk defined as

$$
\operatorname{CVaR}_{\alpha}(z)=\mathbb{E}\left(z \mid z>\operatorname{VaR}_{\alpha}(z)\right),
$$

where $\operatorname{VaR}_{\alpha}(z)$ is the value-at-risk of $z$, that is $F_{Z}^{-1}(\alpha)$.

The following equivalent SSD efficiency criterion holds due to Definition 6:

$$
\operatorname{CVaR}_{\alpha}\left(-Y_{1}\right) \leq \operatorname{CVaR}_{\alpha}\left(-Y_{2}\right), \forall \alpha \in[0,1] \Longleftrightarrow Y_{1} \operatorname{SSD} \text { dominates } Y_{2} .
$$

Employing an equivalent formulation of CVaR derived in Rockafellar and Uryasev (2002)

$$
\operatorname{CVaR}_{\alpha}(Y)=\min _{a \in \mathbb{R}}\left\{a+\frac{1}{1-\alpha} \mathbb{E} \max (Y-a, 0)\right\},
$$

they propose the following linear programming test ${ }^{8}$.

\footnotetext{
${ }^{8}$ The inverse SD constraints, including those based on CVaR and used in Kopa and Chovanec (2008), were developed earlier in Dentcheva and Ruszczyński (2006-a). However, the linear programming test (20) was suggested in Kopa and Chovanec (2008).
} 


$$
\begin{array}{rlrl}
D^{*}(y) & =\max \sum_{k=1}^{m} D_{k} & & \\
\text { s.t. } \operatorname{CVaR}_{\frac{k-1}{m}}(-y)-b_{k}-\frac{\sum_{t=1}^{m} w_{k}^{t}}{m-k+1} & \geq D_{k}, & k=1, \ldots, m \\
w_{k}^{t} & \geq-(X \lambda)_{t}-b_{k}, & t, k & =1, \ldots, m \\
w_{k}^{t} & \geq 0, & t, k & =1, \ldots, m \\
D_{k} & \geq 0, \\
\lambda & \in \Lambda & k & =1, \ldots, m
\end{array}
$$

If $D^{*}(y)>0$, then $y$ is SSD inefficient, the optimal allocation $X \lambda^{*}$ dominates $y$ and $X \lambda^{*}$ is $\operatorname{SSD}$ efficient. Otherwise $D^{*}(y)=0$ and $y$ is SSD efficient.

Substituting the explicit expression for CVaR into (20) gives us the following formulation (we denote $y^{[k]}$ the $k$-th largest, and $y^{(k)}$ the $k$-th smallest element of $y)$.

$$
\begin{array}{rlrl}
D^{*}(y) & =\max \sum_{k=1}^{m} D_{k} & \\
\text { s.t. } \frac{-\sum_{i=k}^{m} y^{[i]}}{m-k+1}-b_{k}-\frac{\sum_{t=1}^{m} w_{k}^{t}}{m-k+1} & \geq D_{k}, & k=1, \ldots, m \\
w_{k}^{t} & \geq-(X \lambda)_{t}-b_{k}, & t, k=1, \ldots, m \\
w_{k}^{t} & \geq 0, & t, k=1, \ldots, m \\
D_{k} & \geq 0, & k & =1, \ldots, m \\
\lambda & \in \Lambda &
\end{array}
$$

Therefore, the first constraint ensures that the optimal solution $x=X \lambda^{*}$ satisfies

$$
\frac{-\sum_{i=k}^{m} y^{[i]}}{m-k+1}+\frac{\sum_{i=k}^{m} x^{[i]}}{m-k+1} \geq 0, \quad k=1, \ldots, m
$$

and therefore

$$
\sum_{i=k}^{m} x^{[i]} \geq \sum_{i=k}^{m} y^{[i]}, \text { hence } \sum_{i=1}^{k} x^{(i)} \geq \sum_{i=1}^{k} y^{(i)}, \quad k=1, \ldots, m
$$


which guarantees dominance of $x$ over $y$ by Definition 7 .

Program (21) comprises both necessary and sufficient condition in one linear program. However, any necessity test can be used at a pre-processing stage to identify inefficiency prior to using (21), for instance Post's (7) or (8). In addition, Kopa and Chovanec (2008) propose another simple test formulated as follows.

$$
\begin{gathered}
d^{*}=\max _{\lambda \in \Lambda} \sum_{k=0}^{m-1} \sum_{j=1}^{n} \lambda_{j}\left(\operatorname{CVaR}_{\frac{k}{m}}(-y)-\operatorname{CVaR}_{\frac{k}{m}}\left(-X^{j}\right)\right) \\
\text { s.t. } \sum_{j=1}^{n} \lambda_{j}\left(\operatorname{CVaR}_{\frac{k}{m}}(-y)-\operatorname{CVaR}_{\frac{k}{m}}\left(-X^{j}\right)\right) \geq 0, \quad k=0, \ldots, m-1
\end{gathered}
$$

which can be rewritten as

$$
\begin{aligned}
& d^{*}=\max _{\lambda \in \Lambda} \sum_{k=0}^{m-1} \sum_{j=1}^{n} \lambda_{j} a_{j k} \\
\text { s.t. } & \sum_{j=1}^{n} \lambda_{j} a_{j k} \geq 0, \quad k=0, \ldots, m-1, \text { with } a_{j k}=\sum_{i=1}^{k} x_{j}^{(i)}-\sum_{i=1}^{k} y^{(i)}
\end{aligned}
$$

Kopa and Chovanec prove that if $d^{*}>0$, then $y$ is SSD inefficient. Moreover, $\lambda^{*}$ is an SSD efficient portfolio that dominates $y$. Note that all $a_{j k}$ can easily be computed a priori, and thus (25) is a linear program with $n$ variables and $m$ constraints. In contrast to Post and Kuosmanen test, it provides an SSD efficient dominating portfolio in case of inefficiency of $y$. Unfortunately, no conclusion can be made concerning the efficiency of $y$ if (25) is infeasible.

In contrast to Kuosmanen (2004) and Dentcheva and Ruszczyński (2003, 2006-b), Kopa and Chovanec use the sum of slacks of CVaRs as the objective function which results in guaranteeing that the optimal portfolio is always SSD efficient and dominates the subject portfolio when the latter is inefficient, with a similar computational complexity.

In summary, (21) offers an attractive linear programming algorithm which comprises the necessary and sufficient condition for SSD efficiency of a given portfolio and provides for an SSD efficient dominating portfolio. The linear program (21) has $m^{2}+2 m+n$ variables and $2 m^{2}+2 m$ inequality constraints. 


\section{Extensions}

Below we consider some extensions to the set of assumptions set out in the previous chapter. We refine the class of preferences and assume away short sales, among others.

\subsection{FSD Efficiency and Optimality}

Due to the important role played by the ordering of portfolio returns in both strong and weak FSD dominance, there is no easy (polynomial complexity) algorithm known to date for identifying the efficiency of even a single given portfolio. Kuosmanen (2004) proposes an MILP-based test for identifying FSD efficiency, whereas Kopa and Post (2009) offer an LP test for the FSD optimality. However, the input data for the latter test can only be obtained by solving an MILP program similar to that of Kuosmanen (2004). Optimization programs with first order stochastic dominance constraints were also studied in Dentcheva and Ruszczyński (2004) and Noyan, Rudolf and Ruszczyński (2006). Post (2003) suggests a seemingly easier LP test for FSD optimality in section V, formula (19). He states that the following condition implies and suffices for FSD optimality of portfolio $y: \theta^{*}(y)=0$, where

$$
\begin{gathered}
\theta^{*}(y)=\min \theta \\
\text { s.t. } \frac{1}{m} \sum_{t=1}^{m} \beta_{t}\left(X \lambda-X_{t i}\right)+\theta \geq 0, \quad i=1, \ldots, n \\
\beta_{i} \geq 1, \quad i=1, \ldots, m-1 \\
\beta_{m}=1, \quad \lambda \in \Lambda
\end{gathered}
$$

Although temptingly simple, this approach turns out to be erroneous. This can be seen on the following example. Consider 3 assets in 2 states: $\mathrm{A}(2,2), \mathrm{B}(1,3)$ and $\mathrm{C}(2.5,1.75)$. The constraints of program (26) for testing FSD efficiency of B become

$$
\left\{\begin{array} { l } 
{ \frac { \beta _ { 1 } ( 1 - 2 ) } { 2 } + \frac { \beta _ { 2 } ( 3 - 2 ) } { 2 } + \theta \geq 0 } \\
{ \frac { \beta _ { 1 } ( 1 - 2 . 5 ) } { 2 } + \frac { \beta _ { 2 } ( 3 - 1 . 7 5 ) } { 2 } + \theta \geq 0 } \\
{ \frac { \beta _ { 1 } ( 1 - 1 ) } { 2 } + \frac { \beta _ { 2 } ( 3 - 3 ) } { 2 } + \theta \geq 0 } \\
{ \beta _ { 1 } \geq 1 , \quad \beta _ { 2 } = 1 }
\end{array} \Rightarrow \left\{\begin{array}{l}
\theta \geq 0.5 \beta_{1}-0.5 \\
\theta \geq 0.75 \beta_{1}-0.625 \\
\theta \geq 0 \\
\beta_{1} \geq 1
\end{array} \Rightarrow \theta^{*}=0.125>0 .\right.\right.
$$


Thus, (26) wrongly classifies B as FSD inefficient.

\subsection{Unrestricted shortsales}

The tests reviewed above assume a convex portfolio possibilities set $\Lambda$. The simplest of these in terms of computational complexity, Post (2003), explicitly assumes restricted short sales ${ }^{9}$. The other methods can handle any polytope $\Lambda$, but are more computationally demanding. Lizyayev (2009) suggests another method particularly efficient in the case of unrestricted short sales, based on decomposition of the matrix of returns and applying gradient optimality conditions similar to those of Dybving and Ross (1980). The method seeks to find an interior point of the set

$$
\left\{\beta \in \mathbb{R}^{m-n}: \quad D\left[\begin{array}{c}
-\left(X_{1}^{\top}\right)^{-1} X_{2}^{\top} \\
I_{m-n}
\end{array}\right] \beta \leq-D\left[\begin{array}{c}
\left(X_{1}^{\top}\right)^{-1} e \\
0_{m-n}
\end{array}\right]\right\}
$$

where $\beta$ is an $(m-n)$-parameter vector, $X_{1}$ are the first $n$ rows of $X$, $X_{2}$ - the rest $(m-n)$ rows $^{10}, D$ is defined via the inverse of the upper triangular $m$-by- $m$ matrix $U_{d}^{m}: D=-\left(U_{d}^{m}\right)^{-1}$.

Program (27) can be equivalently formulated in terms of the slacks of $\beta$ as follows.

$\max \theta$

$$
\begin{gathered}
\text { s.t. }-\left(\left(U_{d}^{n}\right)^{-1}\left(X_{1}^{\top}\right)^{-1} X_{2}^{\top} U_{d}^{m-n}+A\right) \gamma+\theta \leq-D_{1}\left(X_{1}^{\top}\right)^{-1} e, \\
\gamma \in \mathbb{R}^{m-n}, \quad \gamma \geq 0, \quad \theta \geq 0
\end{gathered}
$$

where $A$ is the following $n$-by- $(m-n)$ matrix: $A=\left[\begin{array}{ccc}0 & \cdots & 0 \\ \vdots & & \vdots \\ 0 & \cdots & 0 \\ 1 & \cdots & 1\end{array}\right]$.

Existence of such $\gamma$ (or $\beta=U_{d}^{m-n} \gamma$ ) is a necessary condition for SSD efficiency of the subject portfolio. System (28) is a linear program with $m-n$ variables and $m-n$ constraints (note that as in Post (2003),

\footnotetext{
${ }^{9}$ In fact (7) is only valid for $\Lambda=\left\{\lambda \in \mathbb{R}^{n}: \quad \lambda^{\top} e=1, \lambda \geq 0\right\}$. If $\Lambda$ is another polytope, $X_{t i}$ in (7) should be substituted by the vertices of $\Lambda$.

${ }^{10}$ Assuming without loss of generality that the first $n$ rows of $X$ are linearly independent.
} 
$\gamma$ can be normalized so that $\gamma_{m-n}=\beta_{m-n}=1$ ). Although the method does not find a dominating portfolio, it always identifies if the subject portfolio is efficient and, in such cases, produces a supporting gradient (marginal utility) as a byproduct. The computational advantage of this method becomes particularly eminent when $n$ approaches $m .{ }^{11}$

\section{Comparison of SSD methods}

In Section 3 we have analyzed the major SSD efficiency tests that can be represented as linear programs and therefore can be applied to relatively large real world data. We have shown that the methods differ in terms of the information content of the results and the goal of this chapter is to analyze the computational burden associated with the extra informational outcome of some tests. To make such a comparison objective, we transform each program to the standard form:

$$
\min \left\{c^{\top} x: \quad A x \leq b, x \geq 0\right\} .
$$

A good indicator of the computational complexity of a linear program, at least when a simplex method is applied for solving it, is the average number of non-zero elements in the matrix of constraints at each simplex iteration of changing variables in the basis. This indicator is however difficult to estimate on the basis of the input data. For this reason we follow the Performance World (2009) website and give two indicators for complexity: the size of the $A$ matrix in (29) and the number of non-zeros it contains. For sparse matrices (as in our case) these indicators can be taken to represent, respectively, an upper and a lower bound for the average number of non-zeros in $A$. The table below summarizes the information content of the outcome and the computational complexity of the methods considered in terms of those indicators ${ }^{12}$. All the methods assume away short sales except Lizyayev (2009), which is only applicable to unrestricted short sales.

\footnotetext{
${ }^{11}$ Note however that as $n$ increases, the dimensionality of (28) becomes smaller, but one needs to invert a larger $X_{1}$ prior to solving (28). If $X$ happens to be particularly ill-conditioned, one may rewrite (28) without decomposition as: find $z \in \mathbb{R}^{m}$ such that $X z=e, z \geq 0$. This is a linear program with $m$ variables and $2 m$ constraints, and therefore remains the most efficient method for the case of unbounded $\Lambda$.

${ }^{12}$ The portfolio budget constraint enters every method in the same form and thus was omitted from the complexity analysis for brevitys sake.
} 
苛

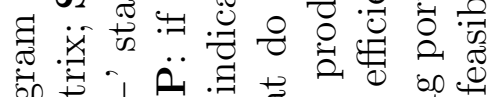

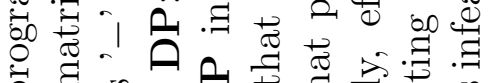

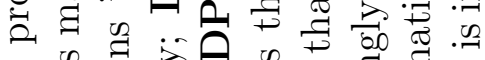

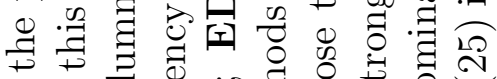
क

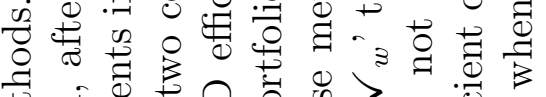

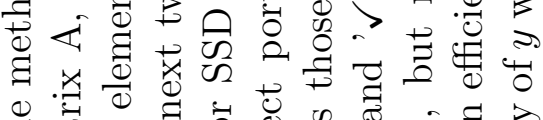

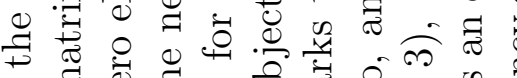
पै घ ज्ञ

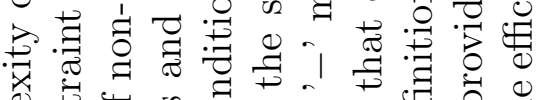

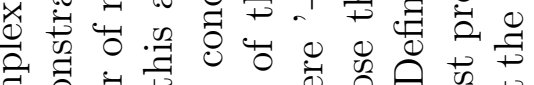
菏

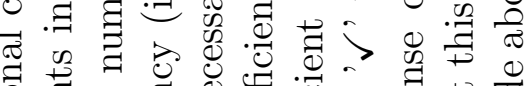

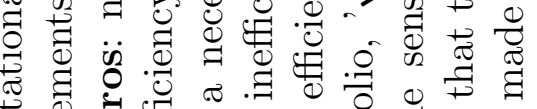

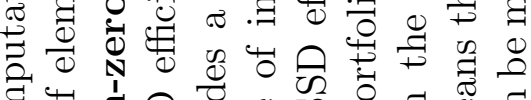

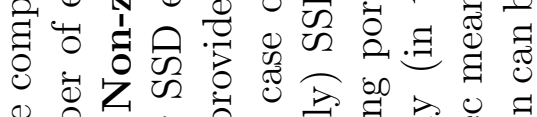

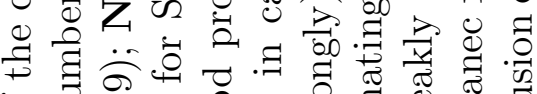

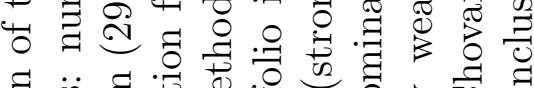

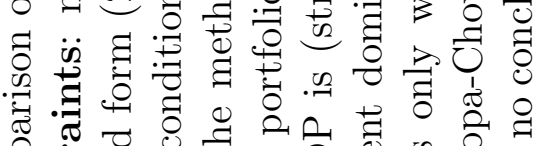

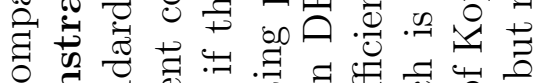
휴

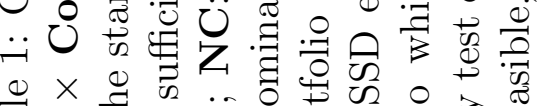

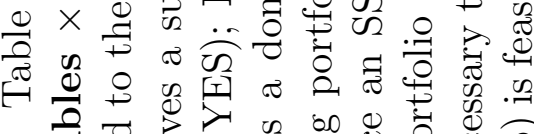
. สี

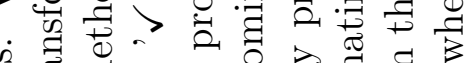

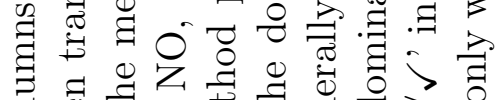

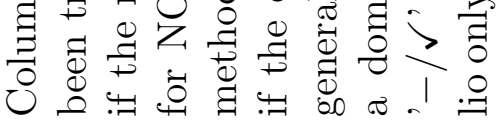

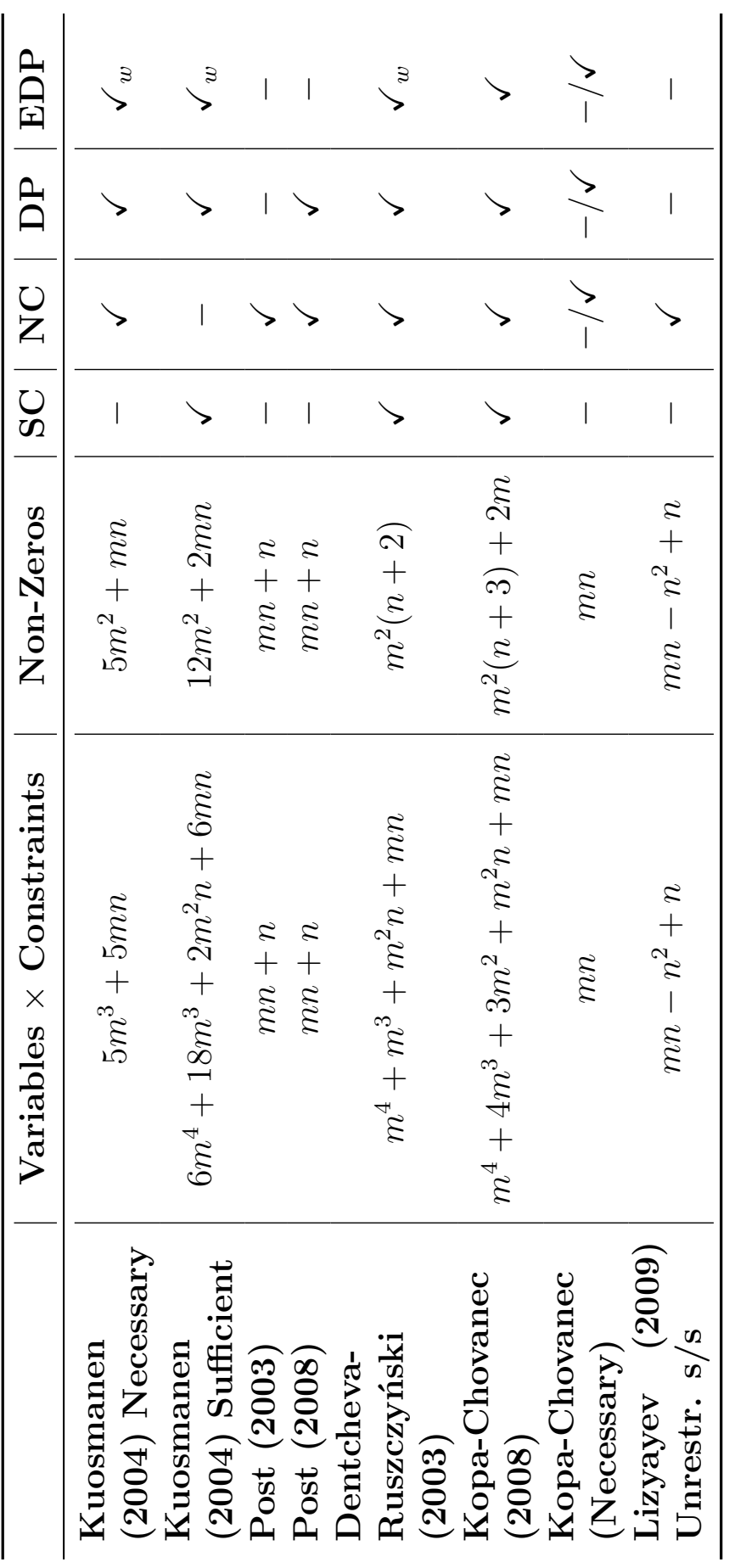


As we can see from Table 1, the methods can be grouped by the information content of the results. The most informative method, Kopa and Chovanec's (21), is also the most computationally demanding. It is the only method that identifies an SSD efficient dominating portfolio with a linear program. Other methods require quadratic programming to identify an SSD efficient dominating portfolio. The Dentcheva and Rudzszynski (2003) and Kuosmanen (2004) necessary tests are identical in terms of the information content of the results. As for the computational complexity, Kuosmanen (2004) is lighter in terms of non-zeros but slightly harder regarding the size of the constraints matrix. A dominating portfolio that is weakly SSD efficient is identified, but the dominating portfolio is not always strongly SSD efficient. The test of Post (2008) is a lot lighter than the previous tests, but it loses in information content. The Kopa and Chovanec (2008) necessary test (25) is slightly lighter than Post (2003) and in some cases identifies an efficient dominating portfolio, although no conclusions can be made concerning the efficiency of $y$ if (25) is infeasible. Both tests are applicable when short sales are restricted. The test of Lizyayev (2009), on the contrary, assumes unrestricted short sales and in this case is the lightest computationally, albeit bearing the minimal information content: just like Post (2003), it only provides a necessary condition for efficiency of the subject portfolio and a sufficient condition for its weak efficiency.

\section{Concluding remarks}

We can summarize the paper as follows. We have taken the various methods of three different schools of thought, some of which are developing independently, without any cross-reference to or interaction with the others, placed them under a common umbrella and analyzed them in a unified methodological framework where both the information content of their results and their computational complexity were compared. We have given a principal classification into three categories based on the definition of SSD efficiency employed in each particular method, but we have also seen that methods from different categories can be grouped according to the content of the results and their computational complexity. For many large- or even medium-size data sets some of the methods may become computationally prohibitive, particularly taking bootstrapping into account when the tests have to be 
repeated many times on similar or even larger data sets simulated from the original distribution. We hope this paper will assist practitioners in finding a desired tradeoff between bearable computational burden and the information content of the results required.

The methods in each school of thought are based on Definitions 1, 6 or 7 . It is remarkable that, although those definitions are proved to be equivalent, the optimization programs corresponding to those definitions substantially differ in terms of their computational complexity, as well as the information content of their outcome.

In addition to classifying and comparing of the methods, we have also corrected some misleading arguments in the literature under consideration and suggested refinements to some of the methods.

\section{References}

[1] ABOUDI R., THON D., Efficient Algorithms for Stochastic Dominance Tests Based on Financial Data, Management Science, April 1994, 40(4), pp. 508-515.

[2] AFRIAT, S., The Construction of a Utility Function from Expenditure Data, Internat. Econom. Rev. 8, 1967, pp. 67-77.

[3] ANDERSON G., Nonparametric Tests of Stochastic Dominance in Income Distributions, Econometrica, 64-5, 1996, pp. 11831193.

[4] ANG J. S., A Note on the E-S Portfolio Selection Model, Journal of Financial and Quantitative Analysis, 1975, 10(5), pp. 849-857.

[5] AnNAert J., van OSSElaer S., VerStraete B., Performance evaluation of portfolio insurance strategies using stochastic dominance criteria, Journal of Banking \& Finance, 33, 2009, pp. $272-280$.

[6] BAWA, V. S.et al, An efficient Algorithm to Determine Stochastic Dominance Admissible Sets, Management Science, July 1979, 25(7), pp. 609-622.

[7] BAWA, V. S. et al, On Determination of Stochastic Dominance Optimal Sets, The Journal of Finance, 40-2 (1985), pp. 417-431. 
[8] BAWA, V. S., Stochastic Dominance: A Research Bibliography, Management Science, 28, 1982, pp. 698-712.

[9] BIXBY R. E., Solving real-world linear programs: A decade and more of progress. Oper. Res. 2002, 50(1) 3-15.

[10] COCHRAne J. H., Asset Pricing, 2nd Ed, Princeton University Press, 2005.

[11] DENTCHEVA D., RUSZCZYŃSKI A., Inverse stochastic dominance constraints and rank dependent expected utility theory, Mathematical Programming, 108 (2006-a), pp. 297-311.

[12] DENTCHEVA D., RUSZCZYŃSKI A., Optimization with Stochastic Dominance Constraints, SIAM Journal on Optimization, 14 (2003) pp. 548-566.

[13] DENTCHEVA D., RUSZCZYŃSKI A., Portfolio Optimization with Stochastic Dominance Constraints, Journal of Banking and Finance, 30/2 (2006-b), pp. 433-451.

[14] DENTCHEVA D., RUSZCZYŃSKI A., Semi-Infinite Probabilistic Optimization: First Order Stochastic Dominance Constraints, Optimization, 53 (2004) pp. 583-601.

[15] DYBVIG P. H., ROSS S. A., Portfolio Efficient Sets. Econometrica, Vol. 50, No. 6. (Nov., 1982), pp. 1525-1546.

[16] FISHBURN P. C., Convex Stochastic Dominance with Continuous Distributions Functions, Journal of Economic Theory, 1974, 7(2), pp. 143-158.

[17] HADAR J., RUSSELL W. R., Rules for Ordering Uncertain Prospects, American Economic Review, 1969, 59, pp. 25-34.

[18] HANOCH G., LEVY H., The efficiency analysis of choices involving risk, Rev. Econom. Stud., 36 (1969), pp. 335-346.

[19] HARDY G. H., LITTLEWOOD J. E., POLYA G., Inequalities, Cambridge University Press, Cambridge, U.K., 1934.

[20] HOGAN W. W., WARREN J. M., Computation of the Efficient Boundary in the E-S Portfolio Selection Model, The Journal of Financial and Quantitative Analysis, September 1972, 7(4), pp. 1881-1896. 
[21] KARLIN S., NOVIKOFF A., Generalized Convex Inequalities, Pacific J. Math., 1963, pp. 1251-1279.

[22] KOPA M., POST T., A Portfolio Optimality Test Based on the First-Order Stochastic Dominance Criterion, The Journal of Financial and Quantitative Analysis, forthcoming.

[23] KOPA M., CHOVANEC P., A Second-Order Stochastic Dominance Portfolio Efficiency Measure, Kybernetika, 44(2), 2008, pp. $243-258$.

[24] KUOSMANEN T., Efficient Diversification According to Stochastic Dominance Criteria, Management Science, Vol. 50, No. 10, 2004, pp. 1390-1406.

[25] KUOSMAnEN T., Stochastic Dominance Efficiency Tests under Diversification, Working Paper W-283, Helsinki School of Economics, 2001.

[26] KUOSMAnen T., Performance measurement and best-practice benchmarking of mutual funds: combining stochastic dominance criteria with data envelopment analysis, Journal of Productivity Analysis, 28(1), 2007, pp. 71-86.

[27] LEVY H., Stochastic Dominance And Expected Utility: Survey And Analysis, Management Science, Vol. 38, No. 4 (1992).

[28] LEVY H., Stochastic Dominance: Investment Decision Making under Uncertainty, Second Edition, Springer Science + Business Media, inc., 2006.

[29] LINTER J., The aggregation of Investor's Diverse Judgments and Preferences in Purely Competitive Security Markets, The Journal of Financial and Quantitative Analysis, Vol. 4, No. 4. (Dec. 1969), pp. 347-400.

[30] LIZYAYEV A. M., Stochastic Dominance: Convexity and Some Efficiency Tests, Tinbergen Institute working paper series, Erasmus University Rotterdam, 09-112/2, 09 December 2009.

[31] LUEDTKE J., New Formulations for Optimization under Stochastic Dominance Constraints, SIAM Journal on Optimization, 2008, Vol. 19 (3), pp. 1433-1450. 
[32] MARKOWITZ H. M., Portfolio Selection, The Journal of Finance, 7 (1952), pp. 77-91

[33] MARKOWITZ H. M., Mean Variance Analysis In Portfolio Choice And Capital Markets, Basil Blackwell, New York, 1978.

[34] MARSHALL A. W., OLKIN I., Inequalities: Theory of Majorization and Its Applications, Academic Press Inc., New York, 1979.

[35] NOYAN N., RUDOLF G. and RUSZCZYŃSKI A., Relaxations of Linear Programming Problems with First Order Stochastic Dominance Constraints, Operations Research Letters, 34 (2006) pp. $653-659$

[36] OGRYCZAK W., RUSZCZYŃSKI A., Dual stochastic dominance and related mean-risk models, SIAM Journal on Optimization, 13 (2002) pp. 60-78.

[37] OGRYCZAK W., RUSZCZYŃSKI A., From stochastic dominance to mean-risk models: Semideviations as risk measures, European Journal of Operational Research, 116 (1999) pp. 3350.

[38] OGRYCZAK W., RUSZCZYŃSKI A., On consistency of stochastic dominance and mean-semideviation models, Mathematical Programming, 89 (2001), pp. 217-232.

[39] PERFORMANCE WORLD (viewed October 2009). GAMS Development Corp. http://www.gamsworld.org/performance/plib/size.htm.

[40] POST T., Empirical Tests for Stochastic Dominance Efficiency, The Journal of Finance, Vol. 58, No. 5. (Oct., 2003), pp. 19051931.

[41] POST T., On the Dual Test for SSD Efficiency: With an Application to Momentum Investment Strategies, European Journal of Operational Research, 185(3), 2008, pp. 1564-1573

[42] POST T., VERSIJP P., Multivariate Tests for Stochastic Dominance Efficiency of a Given Portfolio, JFQA 42-2 (June 2007), pp. $489-516$. 
[43] PRATT J. W., Risk Aversion in the Small and in the Large. Econometrica, Vol. 32, No. 1/2 (Jan. - Apr. 1964), pp. 122-136.

[44] ROCKAFELlAR R. T., URYASEV S., Conditional Value-atRisk for General Loss Distributions, J. Banking Finance, 26 (2002), pp. 1443-1471.

[45] ROCKAFELlaR, R. T., Convex Analysis, Princeton University Press, Princeton, NJ, 1970.

[46] ROSS S. A., Mutual Fund Separation in Financial Theory - The Separating Distributions, Journal of Economic Theory 17, pp. 254-286 (1978).

[47] ROTHSCHILD M., STIGLITZ J., Increasing Risk. I. A definition, J. Econ. Theory, 2 (1970), pp. 225-243.

[48] ROTHSCHILD M., STIGLITZ J., Increasing Risk. II. Its economic consequences, J. Econ. Theory, 3 (1971), pp. 66-84.

[49] RUBInStein M., An Aggregation Theorem For Securities Markets. Journal of Financial Economics I (1974) pp. 225-244.

[50] RUDOLF G., RUSZCZYŃSKI A., Optimization Problems with Second Order Stochastic Dominance Constraints: Duality, Compact Formulations, and Cut Generation Methods, SIAM J. on Optimization, 19(3), 2008, pp. 1326-1343.

[51] RUSZCZYŃSKI A., VANDERBEI R., Frontiers of Stochastically Nondominated Portfolios, Econometrica, 71 (2003), pp. 12871297.

[52] SHALIT H., YITZHAKI S., Mean-Gini, Portfolio Theory, and the Pricing of Risky Assets, The Journal of Finance, December 1984, 39(5), pp. 1449-1468.

[53] SHALIT H., YITZHAKI S., Marginal Conditional Stochastic Dominance, Management Science, May 1994, 40(5), pp. 670-684.

[54] STRASSEN V., The Existence of Probability Measures with Given Marginals, The Annals of Mathematical Statistics, Vol. 36, No. 2 (Apr., 1965), pp. 423-439 
[55] VERSIJP P., Advances in the use of stochastic dominance in asset pricing, Tinbergen Institute PhD Thesis \# 407, Thela Thesis, 2007.

[56] VICKSON R. G., Stochastic Dominance Tests for Decreasing Absolute Risk Aversion. I: Discrete Random Variables, Management Science, 21/12, (Aug., 1975), pp. 1438-1446.

[57] VICKSON R. G., Stochastic Dominance Tests for Decreasing Absolute Risk-Aversion II: General Random Variables, Management Science, 23/5 (Jan., 1977), pp. 478-489. 